\title{
Informarse en el smartphone: estrategias de lectura transmedia por parte de jóvenes universitarios del Aglomerado Gran Buenos Aires*
}

\section{Francisco Javier Albarello ${ }^{1}$}

Recibido: 22/06/2019

Aprobado por pares: 15/08/2019
Enviado a pares: 22/06/2019

Aceptado: 31/08/2019

DOI: 10.5294/pacla.2020.23.3.1

Para citar este artículo/to reference this article/para citar este artigo

Albarello, F. J. (2020). Informarse en el smartphone: estrategias de lectura transmedia por parte de jóvenes universitarios del Aglomerado Gran Buenos Aires. Palabra Clave, 23(3), e2331. https://doi.org/10.5294/pacla.2020.23.3.1

\section{Resumen}

El consumo de noticias se ha transformado sensiblemente en los últimos años, en virtud de la multiplicación de dispositivos a través de los cuales se accede a la información, los cambios en los modos de contar las noticias y la creciente importancia del software en la gestión de la información. El smartphone es el principal medio de acceso a las noticias por parte de los jóvenes universitarios del Aglomerado Gran Buenos Aires, quienes despliegan estrategias de lectura particulares a la hora de informarse. Los jóvenes navegan aprovechando la especificidad que le ofrece cada dispositivo en el modo de presentar las noticias y lo mejor de cada uno. En ese sentido, la "lectura transmedia" parece ser la forma que asume la relación que se establece con esos contenidos, puesto que los relatos noticiosos se despliegan en múltiples plataformas y se completan con la participación activa de los usuarios, quienes ponen en diálogo esas distintas plataformas, comparten

\footnotetext{
* Artículo financiado por la Universidad Austral, Vicerrectorado de Investigación. Proyecto de investigación: “El consumo transmedia de noticias por parte de jóvenes universitarios" (código: T18-15).

$1 \triangle$ https://orcid.org/0000-0001-6623-916X. Universidad Austral, Argentina. falbarello@austral.edu.ar
} 
las noticias en las redes sociales y producen contenidos relacionados con sus temas de interés. En esta investigación exploratoria, procuramos describir y analizar las prácticas de consumo de noticias que realizan los jóvenes a través del smartphone mediante 16 entrevistas en profundidad realizadas a estudiantes de diversas universidades del país. Del análisis de las entrevistas se desprende que los jóvenes despliegan estrategias de lectura habituales en las que alternan entre la exposición y la interacción, entre la lectura superficial y la lectura profunda, y entre la búsqueda de información y entretenimiento.

\section{Palabras clave (Fuente: tesauro de la Unesco)}

Smartphone; teléfono inteligente; consumo; consumidor; lectura; noticias; jóvenes; transmedia. 


\section{Getting Information from the Smartphone: Transmedia Reading Strategies by Young University Students from the Aglomerado Gran Buenos Aires}

\section{Abstract}

News consumption has transformed significantly in recent years due to the multiple devices through which information is accessed, changes in how news are told, and the growing importance of software in information management. The smartphone is the main source of news for young university students from the Aglomerado Gran Buenos Aires, who employ particular reading strategies when it comes to getting information. Young people surf the internet taking advantage of the specificity and best features offered by each device in carrying the news. Thus, "transmedia reading" seems to be the form taken by the relationship established with these contents since news stories are displayed on multiple platforms and complemented by the active participation of users. The latter cause such platforms to interact with each other, share the news on social media, and produce content related to their interests. This exploratory research attempts to describe and discuss the youth's news consumption practices using the smartphone that were collected through 16 in-depth interviews with students from various universities in the country. From the analysis of the interviews, we deduce that young people follow usual reading strategies ranging between exposure and interaction, between intensive and extensive reading, and between information seeking and entertainment.

\section{Keywords (Source: Unesco Thesaurus)}

Smartphone; consumption; consumer; reading; news; youth; transmedia.

* Article funded by the Office of the Vice President for Research, Universidad Austral. Research project titled "El consumo transmedia de noticias por parte de jóvenes universitarios" (code: T18-15). 


\section{Estar informado pelo smartphone: estratégias de leitura transmídia por parte de jovens universitários do Aglomerado Gran Buenos Aires}

\section{Resumo}

O consumo de notícias tem se transformado sensivelmente nos últimos anos, devido à multiplicação de dispositivos pelos quais se tem acesso à informação, às mudanças nos modos de contar as notícias e à crescente importância do software na gestão da informação. O smartphone é o principal meio de acesso às notícias por parte dos jovens universitários do Aglomerado Gran Buenos Aires, Argentina, os quais desenvolvem estratégias de leitura particulares no momento de se informar. Os jovens "navegam" aproveitando a especificidade que cada dispositivo lhes oferece no modo de apresentar as notícias e o melhor de cada um. Nesse sentido, a leitura transmídia parece ser a forma que a relação que é estabelecida com esses conteúdos assume, visto que os relatos das notícias são desenvolvidos em múltiplas plataformas e são completados com a participação ativa dos usuários, os quais colocam em diálogo essas diferentes plataformas, compartilham as notícias nas redes sociais e produzem conteúdos relacionados com seus temas de interesse. Nesta pesquisa exploratória, pretende-se descrever e analisar as práticas de consumo de notícias que os jovens realizam em seus telefones por meio de 16 entrevistas em profundidade realizadas a estudantes de diversas universidades do país. Da análise das entrevistas, conclui-se que os jovens desenvolvem estratégias de leitura habituais nas quais alternam entre a exposição e a interação, entre a leitura superficial e a leitura profunda, e entre a busca de informação e o entretenimento.

\section{Palavras-chave (Fonte: tesauro da Unesco)}

Smartphone; consumo; consumidor; leitura; notícias; juventude; transmídia.

\footnotetext{
* Artigo financiado pela Universidad Austral, vice-reitorado de pesquisa. Projeto de pesquisa intitulado "O consumo transmídia de notícias por parte de jovens universitários" (código: T18-15).
} 


\section{Introducción}

La revolución de las tecnologías de la información y de la comunicación (TIC) de las últimas décadas del siglo XX ha penetrado todas las áreas de la vida social (Castells, 1998), y la forma en que se produce, circula y consume la información no ha sido la excepción. Los medios tradicionales buscan adaptarse al nuevo ecosistema en que las audiencias asumen un rol más activo en las formas de consumir información, ya que tienen una mayor cantidad de canales de acceso, a lo que se suma la posibilidad que le otorgan las tecnologías de producir y compartir información a una velocidad tal que dificulta la posibilidad del análisis y el chequeo de datos. En las primeras décadas del siglo XXI, el acceso a la información está cada vez más mediado por el software que se ha convertido en nuestra interfaz con el mundo (Manovich, 2013) y las plataformas (Fernández, 2018; Srniceck, 2018; Van Dijck, Poell \& De Waal, 2018) que explotan comercialmente la información que le brindan sus usuarios y adquieren una relevancia cada vez mayor en su competencia feroz para captar la atención del lector.

En este contexto, el consumo de noticias se está transformando. En primer lugar, por el papel que desempeñan los medios tradicionales en el ecosistema digital: si bien la prensa diaria sigue marcando agenda en los temas de interés, es claro que su influencia sobre las audiencias es relativa, dado que estas generan rutinas de consumo alejadas de la propuesta de los medios, crean comunidades en las cuales comparten noticias - particularmente a través de las redes sociales - y les otorgan a estas noticias significados que tienen más que ver con sus propios intereses que con los que les proponen los medios. En ese sentido, las redes sociales compiten con los medios informativos en su función de filtrado de la información y el público reparte su credibilidad entre unos y otros, lo que pone en crisis el modelo de negocios del periodismo, que busca el modo de monetizar sus productos en el desafiante ecosistema de las plataformas sociales. En segundo lugar, el contexto descrito nos obliga a preguntarnos hoy qué es noticia. Para Martini (2004, p. 117), la noticia es "la construcción periodística de un acontecimiento", y asociados a este concepto quedaron establecidos los criterios de noticiabilidad, entre ellos, su carácter novedoso. 
Ahora bien, esta definición de noticia está estrechamente vinculada con el papel social que desempeñó el periodismo desde la revolución industrial, dado que en un contexto de escasez de información los medios eran los intermediarios entre ella y el público, considerado como masa (Lima, 2012 , p. 275). Siguiendo esta lógica, la noticia asume la forma de un producto de consumo masivo (Hirst, 2011, p. 69). ¿Qué sucede en la actualidad en un contexto de exceso de información? ¿Es lo mismo una noticia elaborada por un profesional de un medio periodístico que un tuit de un usuario que cuenta lo que vio en la calle? Claramente, el concepto de noticia se está transformando, puesto que no es más un atributo exclusivo de profesionales, sino una manera de contar el mundo por parte de ciudadanos que, en virtud de un acentuado proceso de "amateurización masiva" (Shirky, 2008), tienen en sus manos, como nunca antes, herramientas fáciles de usar que permiten hacerlo en todo momento y en todo lugar. Por último, estas mismas herramientas digitales, en manos de usuarios deseosos de compartir contenido, ofrecen la posibilidad de remezclar esos contenidos y producir nuevas formas narrativas en que se mezclan lo real con la ficción y la información con el entretenimiento, difuminan las barreras que antes separaban los géneros y producen hibridaciones que escapan a las clasificaciones tradicionales y a las posibilidades de control por parte de la industria mediática.

En este artículo, se presentará, en primer lugar, el marco teórico de la investigación, en que se caracterizará la manera como se lee en la actualidad desde la perspectiva del usuario o lector, y no tanto de los dispositivos donde tiene lugar. En ese sentido, se analizará el fenómeno del consumo de noticias desde la perspectiva de la ecología de los medios y las narrativas transmedia. Luego de las aclaraciones metodológicas, se presentarán los hallazgos de esta investigación exploratoria con la intención de establecer y caracterizar algunas estrategias de lectura transmedia que llevan adelante los jóvenes universitarios a la hora de informarse, estrategias en las que ponen en juego los diversos medios en busca de sacar el mejor provecho de cada uno de ellos. Finalmente, en las conclusiones, se abrirán algunas líneas de debate y se comentarán las limitaciones de este estudio. 


\section{Lectura transmedia en el ecosistema digital}

Scolari (2013) define las narrativas transmedia como "un tipo de relato donde la historia se despliega a través de múltiples medios y plataformas de comunicación, y en el cual una parte de los consumidores asume un rol activo en ese proceso de expansión" (p. 46). El rol que asumen los consumidores es mucho más activo que en el pasado, puesto que no solo se trata de consumir o ver, sino de participar y crear. En ese sentido, las narrativas transmedia pueden ser la respuesta a la hiperfragmentación de la audiencia, ya no a partir de un medio particular sino alrededor de un relato (Scolari, 2013, p. 222). Jenkins, quien acuñó el concepto en 2003, se pregunta ¿qué es lo que cada medio hace mejor?, y plantea que no se trata del mismo contenido llevado a otra plataforma, sino que este asume una forma distinta de acuerdo con el medio o plataforma donde se encarna (Jenkins, 2003). Asimismo, las audiencias conocen esas características particulares y buscan aprovecharlas cuando utilizan esos medios. El contenido ya no está meramente empaquetado en una "tecnología de distribución" (Jenkins, 2008) y entregado a un consumidor modelo de acuerdo con un pacto de lectura particular (Scolari, 2004), sino que se ha "desatado", se ha convertido en flujo, y presenta un aspecto distinto y personalizado de acuerdo con la performance de software (Manovich, 2013), que tiene lugar en el dispositivo a través del cual accede el usuario (Albarello, 2017). Estas transformaciones no se limitan a los medios digitales, sino que afectan a todo el ecosistema mediático. Desde la perspectiva de la ecología de los medios (McLuhan, 1994; Ong, 1994; Postman, 1992), Scolari (2015) explica que los medios coevolucionan tanto en su relación con los otros medios — podemos ver, por ejemplo, cómo la TV se está adaptando al consumo audiovisual online que ofrece Netflix - como en su relación con los usuarios, y así producir nuevas prácticas de consumo y pactos de lectura, que tienen lugar en el espacio de negociación de sentido que es la interfaz (Scolari, 2018). Una característica saliente de esta relación con los usuarios es que los medios generan un ambiente o entorno sensorial que pasa desapercibido para los usuarios y que condiciona el contacto de este con el mundo que lo rodea (Scolari, 2015).

Desde estas vertientes teóricas, es posible pensar, entonces, en el desarrollo de un concepto, lectura transmedia, que dé cuenta del complejo fenó- 
meno de la recepción o del consumo en épocas de convergencia mediática. La lectura transmedia es: "Un tipo de lectura inclusiva, multimodal, diversa, de todo tipo de textos — escritos, visuales, sonoros, lúdicos-y de soportes, que a su vez se mezcla o hibrida con las prácticas de producción o prosumo del lector" (Albarello, 2019, p. 166).

Podemos encontrar algunos antecedentes de lectura transmedia en conceptos tales como translector (Scolari, 2016), consumo transmedia (Ilhan, Kozinets \& Otnes, 2013), lectoespectador (Mora, 2012), internauta (GarcíaCanclini, 2007) e hiperlector (Burbules, 1997). Todos ellos comparten la idea de un lector que "lee/navega" (Albarello, 2011) que toma decisiones constantemente a través de diversos caminos posibles de lectura. Asimismo, Jenkins (2009) propone el concepto de navegación transmedia (transmedia navigation) que consiste en la habilidad de seguir el flujo de historias e información a través de múltiples modalidades. El adjetivo transmedia añade aquí lo específico de este momento de la evolución de los medios: la convivencia de una diversidad de plataformas de consumo y producción de contenidos y lenguajes, que complejiza y enriquece aún más la experiencia de lectura. Esto tiene lugar principalmente a través de "tecnologías relacionales” (Aguado, Feijóo y Martínez, 2013, p. 65), como los smartphones y las tabletas, puesto que se insertan en los intercambios que los usuarios hacen en sus comunidades de interés que son las redes sociales. Las mediciones más recientes sobre consumo de noticias a nivel mundial indican que el acceso a estas a través del smartphone se ha duplicado en los últimos seis años, y ha llegado a un $64 \%$ de usuarios en España y un $56 \%$ en los Estados Unidos (Reuters Institute, 2018). En consonancia con estos resultados, según datos de We Are Social correspondientes a octubre de 2018, el tráfico web a nivel mundial está liderado por los teléfonos celulares (51\%) sobre las computadoras de escritorio $(44,1 \%)$ y las tabletas $(4,2 \%)$. Finalmente, de acuerdo con el informe de la consultora Comscore de 2018, hay una prevalencia de un $46 \%$ a nivel global del "usuario multiplataforma" (el que utiliza computadoras de escritorio, smartphones y tabletas), pero el smartphone duplica a la computadora de escritorio en minutos de uso, a la vez que hay un crecimiento importante de la audiencia "solo móvil", y el $80 \%$ de los usuarios de todo el mundo acceden a las noticias a través de este medio. 
En resumen, en el actual ecosistema mediático, si bien los usuarios cuentan con una diversidad de opciones para acceder a las noticias, el predominio creciente del smartphone está dando forma a la relación con las noticias. Sin embargo, esto no quiere decir que los medios tradicionales, como la prensa gráfica, la radio y la TV desaparezcan, sino que — como veremos más adelante- se adaptan y evolucionan para seguir conviviendo en un sistema interdependiente cada vez más vasto de opciones comunicacionales (Fidler, 1998).

\section{Estrategias de lectura en las pantallas}

Aun ante la diversidad de dispositivos en los que se ofrece el texto en la actualidad, es el lector quien toma las principales decisiones sobre qué leer, cuándo leer y cómo leer. Desde la perspectiva de la conversación textual, Bettetini (1986) sostiene que es el lector quien pone en juego su propio proyecto de interacción con el texto, o mejor, de acuerdo con el modelo semio-cognitivo (Scolari, 2004), es el destinatario empírico el que responde al simulacro de usuario que aparece instalado en el texto con su propuesta de interacción. Precisamente, el proyecto o propuesta de interacción que pone en juego el destinatario empírico se traduce en una estrategia de lectura. Podemos afirmar con cierta seguridad que, cuando nos enfrentamos con un texto (escrito, audiovisual, digital, etc.), siempre pondremos en juego alguna o algunas estrategias. En otras palabras, no leemos sin desplegar una estrategia de lectura, la cual puede modificarse durante el trayecto. En palabras de Bettetini (1986), diríamos que no hay lectura sin proyecto de interacción. Van Dijk (1989), en tanto, apela a la metáfora del ajedrez para explicar el funcionamiento de las estrategias de lectura, y sostiene que para ganarle al otro el participante no solo deberá jugar correctamente al ajedrez observando las reglas establecidas, sino que desarrollará las mejores estrategias para hacerle jaque mate al otro (p. 180). Podemos decir, entonces, que las reglas de lectura o de consumo en cada dispositivo están dictadas en gran medida por las convenciones sociales (sobre qué es leer o sobre los géneros, por ejemplo), pero también por las posibilidades de navegación que ofrece la interfaz. Por ejemplo, podemos decir que las reglas de lectura que ofrece el libro o el diario impreso promueven la lectura lineal y concentra$\mathrm{da}$, mientras que la lectura en la pantalla propende a una lectura superficial, multilineal y en simultáneo con otras tareas. Sin embargo, el lector puede 
desplegar diferentes estrategias de lectura, incluso subvirtiendo las reglas que ofrece el dispositivo, cuando decide, por ejemplo, no leer linealmente un libro impreso o cuando elige hacerlo linealmente en la pantalla. Siguiendo a Foltz (1996), las estrategias de lectura están determinadas por una variedad de factores, que incluyen el conocimiento del lector sobre el tema, las metas del lector, sus habilidades y las características del texto (p. 120), en tanto que para Chartier y Hébrard (2002, p. 200) los modos de leer varían primero en función de las finalidades de cada lectura. Del mismo modo, cuando Burbules (1997) proponía el concepto de hiperlector para diferenciarlo de los "usuarios" y los "navegadores", sostenía que no se trataba de distintos grupos de personas, sino de enfoques diferentes de las formas de abordar los sistemas hipertextuales. Es más: en función de su objetivo de lectura, el usuario podía adoptar alguno de estos enfoques, o todos ellos, incluso en una misma sesión.

Hasta aquí hemos hablado de estrategias de lectura y de lectura transmedia en general: ¿qué tienen de específicos estos conceptos cuando hablamos del consumo de noticias? Michelstein \& Boczkowski (2010) sostienen que las investigaciones sobre el consumo online de noticias no ha diferido sustancialmente del modo en que se ha estudiado el consumo de noticias en los medios tradicionales. Esto ha llevado - siguiendo a los autores- a dos posiciones respecto del consumo de noticias: por un lado, los que sostienen que el consumo online ha desplazado el consumo de noticias de los medios tradicionales, y por otro, los que afirman que lo ha complementado. Respecto de los primeros, Michelestein \& Bockzkowski mencionan los trabajos de autores que sostienen que la utilización de los medios tradicionales ha decrecido por el uso de la web (Gentzkow, 2007; Gunter, Russell, Withey \& Nicholas, 2003; Kaye \& Johnson, 2003; Lin, Salwen, Garrison $\&$ Driscoll, 2005). En cambio, aquellos que sostienen la idea de la complementación hablan de las preferencias del usuario por utilizar uno u otro medio de acuerdo con sus intereses y los contenidos que requiere (Chan \& Leung, 2005; Dutta-Bergman, 2004; Flavián \& Gurrea, 2007; Flanagin \& Metzger, 2001; Hujanen \& Pietikainen, 2004; Kayany \& Yelsma, 2000; Lin, Salwen, Garrison \& Driscoll, 2005; Livingstone, 2004; Livingstone \& Markham, 2008; Nguyen \& Western, 2007; Sheehan, 2002). En el apogeo de los medios masivos, en el cual la producción y circulación de noti- 
cias obedecía al modelo heredado de la revolución industrial (Hirst, 2011, p. 69), teorías como la de "usos y gratificaciones" (Katz, 1974) ya ponían el acento en las motivaciones que tenía el público para elegir determinados medios con el objeto de informarse, divertirse o relacionarse con otras personas (McQuail, 1969). Posteriormente, investigaciones como la de LaRose, Mastro \& Eastin (2001) procuraron actualizar la vigencia de la teoría de los usos y las gratificaciones en el nuevo ecosistema mediático, ya no dominado por la TV sino por internet.

Por el contrario, desde la perspectiva de la teoría del nicho y en la ecología de los medios, Dimmick, Feaster \& Hoplamazian (2010) sostienen que, pese al avance de las tecnologías digitales, los medios broadcasting ocupan su nicho tradicional con pocas evidencias de desplazamiento, mientras que las noticias en los dispositivos móviles ocupan el nicho de los tiempos intersticiales. Esta hipótesis se ve luego refutada con el trabajo de Boczkowski, Michelstein \& Matassi (2018), quienes sostienen que el "consumo incidental” de noticias, que tiene lugar mientras se está haciendo uso de las redes sociales $\mathrm{u}$ otras aplicaciones en los espacios de los desplazamientos o en momentos de ocio intersticiales (Igarza, 2009; Lyons, 2012), se ha movido desde la periferia hasta el centro de los repertorios de prácticas de información online, particularmente por parte de los jóvenes. Luego de realizar entrevistas en profundidad a 50 informantes de 18 a 29 años en Argentina, Boczkowski et al. (2018) concluyen que este tipo de consumo de noticias implica patrones de lectura breves y fragmentados, una recontextualización y pérdida de jerarquía de los medios informativos y la coexistencia de tres tipos de filtrado: editorial, algorítmico y social.

La oposición entre los efectos de desplazamiento y complementación en los estudios sobre el consumo de noticias, siguiendo a Michelstein \& Bockzkowski (2010), ha tendido a separar artificialmente a los tipos de medios -impresos, broadcasting y online - y sus efectos, en lugar de concebirlos como un todo integrado. Esto ha llevado a ignorar la interpenetración múltiple del consumo de noticias en los medios. En esa línea, la investigación del comportamiento multiplataforma de las audiencias busca cerrar esa brecha. Taneja, Webster, Malthouse \& Ksiazek (2012) analizan el consumo a través de las plataformas (cross-platform consumption), en busca de identi- 
ficar aquellos "repertorios" que llevan adelante los usuarios e integrar el papel de las prácticas sociales y de los rasgos individuales en la explicación de esos patrones, es decir, basados más en el usuario (user-defined media repertoires) que en las características de los medios. Habiendo investigado a 495 informantes estadounidenses a quienes siguieron durante todo un día para conocer sus hábitos informativos, los autores concluyeron que, en la medida en que tienen más opciones de medios para elegir, la gente hace frente a esa abundancia de suministros y crea repertorios de uso selectivo de esos medios, los cuales están influidos tanto por el contexto social en el cual el medio es utilizado como por su vida cotidiana: los usuarios tienden a incrustar ese consumo multiplataforma en sus actividades diarias - trabajo, estudio, ocio, desplazamientos, descanso- - y esos repertorios se transforman en acciones rutinarias que los usuarios activan y replican en sus prácticas sociales (Taneja et al., 2012). El carácter rutinario de los repertorios de consumo de medios se relaciona con el concepto de marco (frame) o guion (script) que proviene de la psicología cognitiva (Van Dijk, 1989, p. 185) y que sirve para explicar aquellas estructuras de pensamiento estereotipadas que los usuarios tienden a aplicar a nuevas situaciones que tienen similitudes con viejos hábitos, como informarse (Scolari, 2004).

La lectura transmedia que aquí proponemos se ubica en esta línea del consumo multiplataforma, porque pretende dar cuenta de las complejas estrategias de consumo de noticias - repertorios - que llevan adelante los jóvenes habitualmente, en las cuales ponen en diálogo a los distintos medios - los complementan - y a la vez solapan unos medios con otros - los desplazan - en acuerdo con sus intereses, sus necesidades, el momento y lugar donde se encuentren, y también el dispositivo del que disponen en ese momento y lugar. En este estudio, el consumo de noticias busca entenderse como una práctica de lectura transmedia, en la cual los jóvenes universitarios ponen en diálogo distintas plataformas y dispositivos para informarse.

\section{Metodología}

El objetivo general de la investigación aquí presentada es describir las rutinas de consumo de noticias que realizan los jóvenes universitarios en las 
que combinan los distintos medios y plataformas, con el objeto de hallar y caracterizar una serie de estrategias o repertorios de lectura transmedia. Para ello, es necesario conocer qué buscan en cada medio en particular los jóvenes universitarios a la hora de consumir noticias en las distintas plataformas. La hipótesis de la investigación es la siguiente: los jóvenes universitarios, a la hora de informarse, despliegan complejas estrategias de lectura $\mathrm{y}$ de consumo de los distintos medios que tienen a su alcance, y buscan aprovechar lo mejor que desde su perspectiva le ofrece cada uno de ellos.

Para llevar adelante los objetivos planteados y corroborar la hipótesis, se desarrolló una investigación de carácter exploratorio durante 2017. El alcance de esta investigación es descriptivo, ya que "busca especificar propiedades, características y rasgos importantes de cualquier fenómeno que se analice", y también "describe tendencias de un grupo o población" (Hernández, Fernández-Collado y Baptista, 2010, p. 80), en este caso, los jóvenes universitarios. El método elegido es el cualitativo, y la técnica empleada es la de la entrevista focalizada (Valles, 1999, p. 184). La metodología cualitativa busca explicación específica sobre una situación dada (Wimmer y Dominck, 1996, p. 145), en este caso, cómo se informan los jóvenes universitarios. Siguiendo la recomendación de Valles (1999, p. 217) de aproximarse al universo de entrevistados potenciales a través de las fuentes disponibles, se seleccionó una muestra de 16 informantes ( 9 mujeres y 7 varones, de 18 a 24 años) en su carácter de alumnos de carreras universitarias de distintas facultades y casas de estudio de la Ciudad Autónoma de Buenos Aires y el Aglomerado Gran Buenos Aires (AGBA) ${ }^{2}$ y también como usuarios de tecnologías digitales, particularmente el smartphone. El corpus de análisis se limitó a 16 casos debido a la saturación que se produjo en la muestra, es decir, no se encontraban variables significativas o patrones nuevos en los entrevistados. Para analizar los resultados de las entrevistas, se optó por el análisis centrado en temas (Valles, 1999, p. 222): luego de codificar las

2 Las entrevistas fueron realizadas a alumnos regulares de las carreras de Relaciones Públicas, Comunicación Social, Ingeniería, Administración de Empresas, Derecho, Educación, Publicidad, Psicología y Arquitectura de las siguientes universidades: Universidad Austral, Universidad Nacional de Lomas de Zamora, Universidad del Salvador, Universidad Nacional de La Matanza, Universidad de Buenos Aires, Universidad de Ciencias Empresariales y Sociales, Universidad Tecnológica Nacional y Universidad Torcuato Di Tella. Estas casas de estudios se encuentran en lo que se denomina AGBA, que abarca la Ciudad Autónoma de Buenos Aires, 14 partidos de la provincia de Buenos Aires $y$, en forma parcial, otros 18 partidos de dicha provincia. 
transcripciones de las entrevistas con el software Atlas.ti, se establecieron comparaciones y se construyeron categorías explicativas para, finalmente, presentar los resultados en forma integrada a través de una línea o secuencia argumental y narrativa reproduciendo extractos de las entrevistas realizadas a los jóvenes. Las categorías de análisis centrado en temas y que se aplicaron al corpus de entrevistas estuvieron vinculadas con las rutinas informativas que desempeñan los jóvenes, la manera en que ponen en diálogo las características diferentes de los medios y dispositivos que utilizan para informarse, y las estrategias de lectura que desarrollan para perseguir ese objetivo.

En cuanto a las consideraciones éticas de este estudio, en la reproducción de los extractos de las entrevistas, y en acuerdo con los entrevistados, se mantuvo el nombre de pila de los entrevistados y no así el apellido, para preservar su anonimato. El hecho de contar con datos como el nombre, la edad y la carrera que cursan estos permite dar cuenta de la heterogeneidad de la muestra, lo cual hace a una mayor riqueza en los hallazgos del estudio. Dado el tamaño de la muestra, los resultados y las reflexiones que se presentan a continuación deben ser interpretados como una serie de hallazgos iniciales que permiten orientar futuras investigaciones en las que se corroboren estos datos con muestras de mayor envergadura o pertenecientes a otros contextos geográficos.

\section{Análisis y resultados}

Los jóvenes universitarios desarrollan rutinas particulares de consumo de noticias a lo largo del día, y hacen uso de la multiplicidad de medios y dispositivos con que cuentan, principalmente el smartphone. El recorrido que hacen por esos diversos medios a lo largo del día obedece, por un lado, a ciertas características que presentan esos medios al ofrecer la información $y$, por otro, a las estrategias de consumo que ponen en juego para satisfacer sus necesidades informativas. En este apartado, nos detendremos a analizar en qué medida el dispositivo influye en el modo de informarse. Buscaremos caracterizar cierto pacto de lectura que establecen los jóvenes con esos dispositivos. Intentaremos responder por qué y cómo van a buscar en ciertos dispositivos algunos contenidos que no irán a buscar en otros. 
En otras palabras, qué esperan de cada uno de los dispositivos que utilizan para informarse.

\section{Las rutinas informativas de los jóvenes}

Las tecnologías digitales están totalmente integradas a las rutinas de consumo de noticias que desarrollan los jóvenes universitarios. Como veremos en este apartado, el uso del celular hegemoniza y a la vez complementa el uso de otros medios y plataformas para informarse. Es muy difícil establecer un único patrón en los hábitos de consumo de noticias que tienen los jóvenes, pero de esta diversidad podemos extraer algunos rasgos comunes que ayudan a definir cómo llevan adelante su día y cómo se informan a lo largo de este.

Son muy pocos los jóvenes que al comenzar su día lo primero que hacen es encender el televisor. Solo 2 de los 16 entrevistados dijeron utilizar este medio para informarse cuando se despiertan:

Bueno, me levanto y prendo la tele. Mientras voy haciendo todo escucho el noticiero. Cuando salgo, tomo el colectivo, auriculares, a veces escucho un poquito de radio, por lo general no últimamente. (Verónica, comunicación personal, 29 junio 2018)

Lo primero que hago cuando me levanto es prender la televisión. No por gusto sino por ya, porque lo prendo y me acostumbré [...] El noticiero televisivo a la noche no me atrae. A la mañana sí, para estar al tanto. (Tomás, comunicación personal, 22 septiembre 2018)

La TV, a la mañana temprano, antes de salir, está como "ruido de fondo" habitual mientras se desarrollan las actividades previas a la salida del hogar. Sin embargo, la función de la TV de ofrecer la información básica para antes de salir, en otros casos y en forma creciente, es ocupada por el teléfono celular: "A la mañana cuando me levanto agarro el teléfono para chequear clima y transporte. Una vez que llego al transporte sigo chequeando el transporte” (Luz, comunicación personal, 25 julio 2018).

En otros casos, en el inicio del día, los jóvenes consultan en sus teléfonos móviles las novedades en sus redes sociales o eligen leer el diario allí: 
Desayuno y casi siempre que desayuno entro a las redes sociales. Ahora que me pongo a pensar, me informo bastante por redes sociales. Las cuentas en Twitter cuentan noticias [...] en general cuentas de noticias sigo. Y después sobre noticias específicas, medio ambiente, deportivas, cuentas específicas. Snapchat tiene bastante de eso también, entonces veo bastante de deportes [...] la CNN tiene para ver noticias, después espectáculo también. Por ahí entro a la aplicación de La Nación pero un pantallazo de dos minutos antes de salir. Generalmente noticias de política es más adentrado el día. (Tomás, comunicación personal, 25 julio 2018)

Me gusta hacer fiaca en mi cama mirando el celular y ahí me informo, no sé, si tengo mensajes de WhatsApp hablo con mis amigas y me estoy informando. Si miro las redes sociales y hay algo que me interese le presto atención. (Camila, comunicación personal, 17 julio 2018)

El consumo más intenso de noticias se hace, principalmente en forma intersticial en los tiempos de desplazamiento hacia el trabajo o la facultad, momentos en los cuales los jóvenes sacan provecho de las características interactivas que tienen las aplicaciones que usan para informarse:

Por lo general yo cuando [...] yo voy al trabajo, o sea, en transporte público, aprovecho abro La Nación, por lo general, desde el celu 0 Google News que te hace una recopilación de distintos medios [...] Entonces más que nada cuando viajo, después cuando vuelvo del trabajo y en la hora de almuerzo también. (Verónica, comunicación personal, 29 junio 2018)

En muchos casos, la rutina informativa no empieza por los diarios digitales (o sus aplicaciones informativas en el celular), sino por las redes sociales, porque los jóvenes valoran su velocidad y su actualización:

Generalmente empiezo por Twitter a la mañana 0 a la tarde. Siento que es más inmediato, que lo actualizan más seguido. Me acuerdo con el atentado de Londres que me llegó una notificación de Clarín, pero no abrí Clarín, fui a Twitter, que sabía que iba a estar más actualizado. (Catalina, comunicación personal, 24 agosto 2018)

La disponibilidad de aplicaciones sociales e informativas en el mismo dispositivo lleva a hacer un consumo híbrido de redes sociales y noticias. En otras palabras, una cosa va llevando a la otra: 
Veo WhatsApp y leo los diarios. Así como estoy en WhatsApp paso a Instagram y de Insta paso a los diarios. Si es por prioridad siempre leo Infobae, Olé y a veces en La Nación si me lo pone la app como prioritario. (Eduardo, comunicación personal, 29 agosto 2018)

Una particularidad del consumo intersticial de información lo presentan los jóvenes que cuentan con movilidad propia, quienes escuchan radio, y en muchos casos es el único momento del día en que lo hacen:

A la mañana pongo la radio en el auto, el $90 \%$ de las veces. Durante un mes tal vez pongo $60 \%$ la radio y $40 \%$ música mía directamente conecto el celular. (Marcos, comunicación personal, 24 agosto 2018)

Generalmente cuando yo salgo a la mañana a trabajar, salgo muy temprano y escucho la radio. Me pongo al tanto mínimamente de la actualidad [...] No vuelvo a actualizarme hasta el horario de ir a la facultad que me vuelvo a subir al auto y es el momento que tengo para escuchar la radio. (Patricio, comunicación personal, 26 febrero 2018)

Una vez que se llega a destino (la facultad o el trabajo), el consumo de noticias adquiere otras características, ya que convive con las otras actividades que se realizan a la par:

\begin{abstract}
Mientras viajo, voy viendo algunas noticias. Llego al trabajo, me instalo, hago unas cosas del trabajo y busco el diario La Nación y lo tengo abierto todo el día [...] Siempre a la mañana pongo el diario. (Verónica, comunicación personal, 29 junio 2018)

No tengo aplicaciones de los diarios. Nunca me llamó la atención. Me parece más cómodo entrar desde la web, desde mi computadora. Me abro muchas pestañas y voy mirando. (Tomás, comunicación personal, 22 septiembre 2018)
\end{abstract}

Resulta interesante destacar el uso de la expresión "pongo el diario" que hace una de las entrevistadas, emulando a lo que se hace con el televisor, para que las noticias estén allí, disponibles mientras se hace otra cosa en la computadora. A diferencia de destinar un tiempo específico para informarse, aquí el diario o los portales de noticias están abiertos todo el día para acceder a ellos cuando se desee. En tanto, el hábito de leer el diario papel — casi inexistente en estos jóvenes - se asocia con "estar en casa": 
"Después sí a la tarde en casa leo el diario. Leer el diario papel está más relacionado con casa, me siento en una silla y lo leo” (Tomás, comunicación personal, 25 julio 2018).

Es decir, leer el diario papel se asocia con una actividad más tranquila, en la que se destina un tiempo en particular. Ahora bien, los jóvenes hacen una diferencia entre los modos de informarse durante la semana, vinculada con la búsqueda constante de información, y los fines de semana, donde procuran "desconectarse" un poco de esa rutina asociada a los dispositivos digitales:

Durante los fines de semana lo que intento es desconectarme de todo lo que son dispositivos con internet. En la semana estoy constantemente buscando noticias para poder estar informada en el trabajo de las cosas que pasan en el país. (Daira, comunicación personal, 26 febrero 2018)

Es de destacar la expresión que usa la joven: "buscar noticias”, una práctica muy distinta del consumo tradicional de noticias en el que el público se exponía a lo que le ofrecía el medio (televisivo o radial). En este caso, la búsqueda de noticias se presenta como una extensión de la lectura del diario, esta vez, de manera interactiva a través de las aplicaciones o las redes sociales que ofrecen los dispositivos.

Para concluir nuestro recorrido por las rutinas informativas diarias de los jóvenes, hay que destacar que antes de irse a dormir lo habitual es un "pantallazo", un momento breve de consumo de noticias:

A la noche es un pantallazo antes de dormir. Por ahí veo una noticia específica antes de dormir y dejo el teléfono... 0 la compu. (Tomás, comunicación personal, 25 julio 2018)

A la noche trato de desconectarme. Solo pongo cuando llego de la facultad lo que es TN para ver qué pasó, si pasó algo en ese lapso que cursé. Después no más. (Daira, comunicación personal, 26 febrero 2018)

Esta costumbre de hacer un pantallazo por la información antes de dormir para enterarse de lo que sucedió cuando no se estaba conectado es 
similar a lo que sucede con las redes sociales: "Antes de dormir es el momento que más consume la gente. Revisás todo: Facebook, Twitter, Instagram, diarios, Snapchat. Todas las aplicaciones que tengas" (Eduardo, comunicación personal, 29 agosto 2018

Es decir, se busca estar al tanto de lo que sucede — en el mundo, en el país, con sus amigos - casi en un mismo nivel de importancia. Y no es un dato menor que esto sea realizado en un mismo dispositivo: el teléfono celular. Las noticias asumen un aspecto social en el que se alternan las noticias duras con las sociales, lo que sucede lejos con lo inmediato, lo que acontece a los desconocidos y a los cercanos.

\title{
¿Qué es lo que cada medio hace mejor?
}

Un aspecto clave de estos repertorios o estrategias de lectura, como señalamos, es que se transforman en hábitos que se cristalizan e incorporan a la vida cotidiana:

\begin{abstract}
Escuchar radio en el auto, leer noticias en la computadora. Se te genera más un hábito. Es una rutina ya eso. (Tomás, comunicación personal, 22 septiembre 2018)

Es la rutina que te lleva a tener que saber de las cosas que pasan en el día. Es la rutina la que te hace ver a la mañana cómo va a estar el clima para saber cómo tengo que salir de casa y cómo voy a estar vestida en la calle [...] Uno no lo busca, la información llega a vos directamente. (Daira, comunicación personal, 26 febrero 2018)
\end{abstract}

La información llega al usuario sin buscarla, esta es tal vez la expresión que mejor grafica el ecosistema actual de medios en el que la información se ha vuelto parte del ambiente mediático donde se mueven los jóvenes. Las rutinas informativas que despliegan son el precio que se paga por estar metidos dentro de ese ambiente de sobreinformación, y las estrategias que desempeñan, centradas en el interés y la conveniencia, son las formas de lidiar con semejante caudal de datos. En ese contexto, hay en los jóvenes una percepción de disponibilidad mayor de fuentes o medios para acceder a la información, que hace que puedan decidir con más libertad cómo y dónde informarse: 
Me parece que cambió porque ahora tenés más opciones de búsqueda. Uno ya tiene en la mente distintas fuentes y va a buscarlas. Después uno obviamente escucha cuando viaja y está la radio prendida en el colectivo y vas informándote. (Verónica, comunicación personal, 29 junio 2018)

"Uno ya tiene en la mente distintas fuentes y va a buscarlas", con lo que se expone claramente que los jóvenes acceden a las noticias con una predisposición particular y un conocimiento de las marcas informativas que conforman el ecosistema de medios. Los medios - tradicionales y nuevos - conviven en un ecosistema que los jóvenes recorren de un lado al otro en busca de información y entretenimiento, y asociando unos contenidos con otros de acuerdo con sus intereses:

Me pasa mucho que mis amigos más grandes usan mucho más Facebook que Instagram. En Instagram yo sé que son fotos y ahora con la historia y todo eso podés ver el minuto a minuto de tus amigos. Pero también hay muchas cosas de famosos que en Facebook no hay. A mí la tele, por ejemplo, no me llama la atención... los programas de chimentos y eso. Sin embargo, en Instagram veo algo o mis amigas lo comentan por WhatsApp y me meto a mirar. Después, en Facebook... yo siento que sirve para contar un poco más lo que estás haciendo. No es simplemente una foto. Capaz que por Facebook también me comunico más por el chat, por el Messenger, con la gente... que en Instagram no me pasa. (Camila, comunicación personal, 17 julio 2018)

En este ecosistema de medios, los jóvenes en general asocian Twitter con la información instantánea (clima, tránsito, último momento) y también con el celular como dispositivo privilegiado de acceso:

La verdad tengo esa tendencia de irme al diario, pero con el tránsito me voy al Twitter [...] Porque Twitter es más de lo instantáneo, de lo rápido, de lo que pasa ahora. (Verónica, comunicación personal, 29 junio 2018)

Sí, Infobae y La Nación son los principales medios que uso. Pero sí, está en mis ventanas de favoritos de todos los días... entrar al menos una vez al día a Infobae para ver qué pasa. Aunque ahí entro desde la computadora. Desde el celular me informo más con Twitter. Facebook no tiene tanto pero a veces podés encontrar algo. En Twitter leo muchas cosas de noticias. (Tomás, comunicación personal, 22 septiembre 2018) 
Los jóvenes reconocen que cada medio ofrece una experiencia diferente. Hay un pacto de lectura, una expectativa que depositan en cada medio; privilegian la comodidad y el acceso a lo que les interesa y el lugar donde están en ese momento. Se prioriza la noticia o el contenido y luego se piensa en cuál es la plataforma que se lo ofrece mejor:

Cuando consumo un medio sí busco cosas. Cuando consumo tele busco el clima, los accesos. Las noticias que te cuentan en segundos, títulos. En internet me fijo un poco más todo. (Daira, comunicación personal, 26 febrero 2018)

Las redes sociales en el celular de una. El noticiero en la televisión. El diario, ahora en el celular. Pasó del diario papel al celular. (Verónica, comunicación personal, 29 junio 2018)

La diferencia está en que en el móvil tengo acceso más fácil a algunas cosas que no tengo ni en TV ni en compu. A las redes no entro desde mi compu más que Facebook... Insta o Snapchat es solo de teléfono [...] Depende de qué tenga a mano, si es la facu lo voy a ver en el celu, si estoy usando la compu consumo desde ahí. (Tomás, comunicación personal, 25 julio 2018)

Podemos decir que, con el uso cotidiano de los distintos dispositivos, los jóvenes entienden ese pacto de lectura, y conociendo las posibilidades y también las limitaciones de cada plataforma, van a buscar cosas diferentes en cada medio que utilizan:

Creo que cada medio, compu, teléfono, redes sociales o aplicaciones como La Nación y la TV son medios que ofrecen algo de determinada manera. Uno ya sabe cuál es el modo de operar de cada medio, entonces uno lo toma o lo deja. Uno ya sabe, cuando va a escuchar 0 consumir, que se va a tener que ajustar a eso. El teléfono es el más versátil, la TV frente a la compu y al teléfono queda como retrasada. Porque en los otros uno puede buscar lo que sea de la forma que sea. (Tomás, comunicación personal, 25 julio 2018)

Luego, hablando del consumo audiovisual compartido, la computadora presenta sus limitaciones si la comparamos con la TV:

Al ser una pantalla bastante más chica es más incómodo compartir. Me acuerdo de haber visto en mi casa con mis siete hermanos en la pantalla de la computadora y no se podía. Es la atmósfera de cada pantalla. (Catalina, comunicación personal, 24 agosto 2018) 
Cada plataforma tiene su "atmósfera" asociada, crea un ambiente a su alrededor que propone formas concretas de apropiarse del contenido. Mientras que esto sucedía con la pantalla de la computadora, el teléfono celular, que es el dispositivo que más utilizan para informarse, tiene asociadas ciertas características, entre ellas, la interactividad, la actualización constante y la inmediatez:

Lo que permite el móvil es tener acceso a las noticias en tiempo real porque se actualiza permanentemente. No tengo horarios fijos para entrar en La Nación o la BBC, sino que a lo largo del día me voy metiendo a ver si sale algo relevante y me permite estar constantemente al tanto de las noticias. (Tomás, comunicación personal, 25 julio 2018)

P. ¿Hay algo que busques en los dispositivos móviles y no en la televisión, la radio y el papel?

R. Interactividad. 0 sea, que sea rápido. Ponele ¿te gusta el ejemplo del clima? Bueno, quizás tengo que estar veinte, quince minutos, en el noticiero a ver qué pasa y en el celular escribo "clima" y ya está. (Luz, comunicación personal, 25 julio 2018)

Llegados a este punto, resulta necesario establecer algunas reflexiones sobre la relación entre los medios tradicionales y el dispositivo digital, particularmente el smartphone. Según se desprende del discurso de los jóvenes entrevistados, en sus rutinas informativas ellos mezclan, superponen y solapan unos medios con otros, sin reparar si se trata de un medio tradicional — como la TV, la radio o el diario impreso, con sus lenguajes asociados a sus tecnologías de distribución - o un dispositivo digital como el smartphone, que en su carácter de "computadora metamedium" (Manovich, 2013) tiende a emular a los medios tradicionales y a hibridarlos entre sí. El teléfono móvil contiene los medios tradicionales y sus lenguajes (Márquez, 2015), los integra en una misma plataforma y los jóvenes ponen en diálogo estas características aprovechando esta disponibilidad en un mismo aparato que les ofrece conveniencia y rapidez.

\section{Estrategias de lectura transmedia}

En atención a las diferencias que presenta cada medio para informarse y lo que han dicho los jóvenes entrevistados, hemos identificado tres categorías de estrategias de lectura que aquí caracterizaremos como pares opuestos 
pero a la vez complementarios: medios expositivos/medios interactivos, lectura superficial/lectura profunda y entretenimiento/información. Estas estrategias tienden a cristalizarse y repetirse a lo largo del día para lidiar con la información. En la primera de ellas, los jóvenes hacen una clasificación general entre medios expositivos (la radio, la TV) que muestran lo que el medio quiere y frente a la cual asumen una actitud más contemplativa, y medios interactivos (el celular, la computadora, internet, Google), donde ellos asumen una posición más activa en la que buscan la información, la profundizan o se interesan por algo:

En el dispositivo móvil, es mucho más fácil buscar puntual ciertas palabras que te lleven a la noticia o a la información que te interesa específica. En los medios es más de lo que te quieren decir o tratar ellos [...] En la televisión, por ejemplo, opto más por las perspectivas y visión de cada uno de un tema en particular. En el teléfono, prefiero tener la información completa del tema, poder entenderlo y abarcar todos los aspectos. (Agustina, comunicación personal, 29 junio 2018)

Es interesante que esta joven ponga en diálogo la TV y el celular, y los vea como medios que complementan el modo de brindar información: la primera invita a contemplar un espectáculo en el que prevalecen las opiniones de los que allí aparecen; y el segundo es el lugar donde ampliar, buscar y completar esa información de acuerdo con la propia inquietud. Sin embargo, esta diferencia entre medios expositivos y medios interactivos es valorada de manera diferente por este otro joven, quien prefiere los medios interactivos, precisamente, porque le permiten buscar la información que le interesa y no estar expuesto a las opiniones de otros cuando no le interesan:

R. No me gusta leer opiniones, me gusta leer más hechos, y esto pasa en la radio, o no sé, mucha más gente hablando de su opinión y no de sobre lo que pasó, y me interesa más lo que pasó.

P. ¿Y esperás una cosa distinta de cada plataforma en particular?

R. Sí, totalmente, por eso leo esas plataformas, porque sé con lo que me voy a encontrar cuando las leo. (Ignacio, comunicación personal, 25 julio 2018) 
La segunda estrategia de lectura que despliegan los jóvenes tiene que ver con el tema, el interés y el tiempo del que disponen para informarse. En este caso, la oposición es entre una lectura superficial o "pantallazo" y una lectura profunda:

Intento leer todo lo que es internacional, detenidamente, noticias enteras. Lo local es más un pantallazo y lo que suelo leer bastante es la deportiva. Un intermedio entre pantallazo y profundo. Depende la categoría es o más profundo o más pantallazo. Internacional es todo profundo. Está pautado de antemano, si no siento que me paso mucho tiempo. Al diario papel sí le dedico más tiempo. (Tomás, comunicación personal, 25 julio 2018)

Es interesante destacar la utilización el término pantallazo aplicado tradicionalmente a la televisión y que ahora se extiende a las otras pantallas, como las del teléfono móvil. Este uso denota una asociación entre pantallas — lo visual — y superficialidad, mientras que la profundidad aparece asociada con la lectura detenida de la información escrita. Sin embargo, la alternancia entre lectura superficial y lectura profunda no es tanto o exclusivamente una propiedad de los dispositivos como una estrategia que desarrollan los lectores. Como veremos a continuación, la profundización sobre una noticia tiene lugar en la TV:

Siento que el noticiero profundiza o puede llegar a dar un análisis que el celular no te lo da. A pesar de que el espacio en internet es ilimitado, si es una nota que es muy larga no llegás a terminarla. (Catalina, comunicación personal, 24 agosto 2018)

Es decir, si se privilegia la comodidad y la conveniencia, entonces la profundidad está asociada más a la TV —y por ende a la noticia como un producto cerrado y editado por el canal — que al teléfono celular, porque este último implica un compromiso prolongado con la noticia, que no se puede o no se quiere terminar de leer.

La tercera y última estrategia de lectura transmedia obedece a una distinción entre entretenimiento e información, que los jóvenes aplican a los medios de modo diferente: la TV, la radio y las redes sociales (como Facebook e Instagram) están más vinculadas con el entretenimiento, mientras que leer 
el diario (en la computadora o en el celular), consultar las redes sociales como Twitter y buscar noticias están más relacionados con la información:

Las de Facebook no son muy relevantes. En cambio, capaz que sí busco algo en particular, no sé, alguna noticia que me interesó. Que la vi en el noticiero o la escuché. (Natalia, comunicación personal, 25 julio 2018)

Generalmente las redes sociales las uso más a modo informativo social o de mi contexto social más que buscando noticias. No lo suelo usar como una plataforma de noticias. (Patricio, comunicación personal, 26 febrero 2018)

La expresión del "uso informativo social" que emplea este último entrevistado sintetiza claramente cómo las redes sociales son escenario de una hibridación entre la noticia — tradicionalmente asociada a lo que les ocurre a las otras personas, más o menos cercanas al usuario y siguiendo ciertos criterios profesionales de noticiabilidad que sustenta un medio periodístico- y la información, producida por los contactos o amigos del usuario en sus redes sociales. La noticia adquiere nuevos atributos porque la cuentan los amigos que forman parte de sus redes de contactos, pero a la vez se la diferencia de la información social, generada por los usuarios, y la relevancia funciona de un modo distinto para cada una de ellas. En otras palabras, y de acuerdo con las prácticas descritas, noticia es también lo que los jóvenes entienden por noticia. Asimismo, informarse es entretenido, en la medida en que los jóvenes disponen de una oferta inacabable de noticias en un dispositivo que atrapa su atención y que los acompaña durante todo el día, sobre todo en los momentos de ocio en los que eligen informarse.

\section{Discusión y conclusiones}

A la hora de informarse, los jóvenes universitarios del AGBA ponen en diálogo los rasgos diferentes que tienen esos medios para ofrecer información, es decir, los complementan. Al mismo tiempo, se observan claros desplazamientos de los medios tradicionales, que tienden a ocupar un nicho particular en el ecosistema de medios que rodea a los jóvenes, en el medio del cual el consumo intersticial de noticias a través del dispositivo metamedium del smartphone se vuelve central. Este estudio exploratorio permitió identificar 
y describir un repertorio de tres estrategias de lectura transmedia: exposición/interacción, pantallazo/profundo e información/entretenimiento. Lejos de tratarse de esquemas fijos correspondientes a cierta tipología de usuarios, se trata de estrategias que estos ponen en juego en distintos momentos del día, según, principalmente, factores como el interés, la función o el objetivo que persigue esa información, el momento del día, el lugar donde se encuentran y la tecnología disponible en ese momento. Este tipo de consumo multiplataforma tiende a desdibujar las fronteras tradicionales entre los diferentes medios, hibrida los contenidos y diluye el vínculo entre medio y contenido, por lo que genera nuevos pactos de lectura entre el usuario y el sistema a través del espacio de coevolución que representa la interfaz. No se trata meramente de sumar plataformas o medios a la dieta cognitiva de los usuarios: los jóvenes buscan sacar provecho con la intención de extraer lo mejor que para ellos puede ofrecer cada medio. El efecto de desplazamiento se produce en la medida en que los medios tradicionales han perdido su hegemonía y ocupan un nicho específico en el ecosistema mediático. La especificidad que ofrece cada medio para acceder a las noticias es puesta en diálogo con la especificidad de los otros medios y aglutinada en el dispositivo hegemónico del smartphone. Los jóvenes no conciben a los medios por separado, sino que al ponerlos en diálogo dan cuenta de la interpenetración creciente entre los distintos medios. Esta perspectiva de análisis, basada más en las necesidades del usuario que en las características de los medios, permite visibilizar las prácticas sociales que desarrollan los jóvenes con los medios y el peso que allí tienen las características individuales de los usuarios.

Por otra parte, estos repertorios de estrategias de lectura transmedia se transforman en hábitos que - a modo de marcos o frames — los jóvenes tienden a aplicar en forma repetida y alternada en su día a día. La interactividad que ofrece el smartphone, sustentada en el software y en la conexión ubicua a internet, acostumbra a los jóvenes a un vínculo con la información caracterizado por la conveniencia, la comodidad y la inmediatez. Este tipo de relación con la información se contagia a los medios tradicionales, a partir de lo cual los jóvenes van a buscar en ellos determinados tipos de contenidos dentro de sus rutinas informativas. Por tanto, el ecosistema 
de medios se ha visto transformado en su totalidad, y ante la ingente cantidad de opciones disponibles, los jóvenes realizan sus recorridos de lectura sin limitarse a los pactos de lectura de los medios tradicionales sino creando los suyos propios.

Este estudio presenta, al menos dos limitaciones: por un lado, se trata de una investigación de carácter exploratorio que incluye una muestra limitada de entrevistados: 16 jóvenes que asisten a la universidad en el AGBA. Las futuras investigaciones deberían considerar la posibilidad de extrapolar este repertorio de estrategias de lectura transmedia halladas a un universo más amplio de usuarios, valiéndose de metodologías cualitativas y también cuantitativas, lo cual permitiría, no solo encontrar continuidades con las estrategias descritas, sino ampliar este repertorio con otras nuevas. En segundo lugar, dado que el ecosistema mediático cambia constantemente en virtud de las innovaciones tecnológicas, es posible que estas estrategias vayan mutando y multiplicándose de acuerdo con diferentes momentos y contextos sociales y culturales. De todos modos, el enfoque que aquí se propone, en el cual se ponen en diálogo aspectos vinculados con los medios en perspectiva ecológica y evolutiva con los factores relacionados con las necesidades del usuario, permite alejarnos de posiciones dicotómicas o reduccionistas para comprender el complejo panorama del consumo de noticias de la actualidad.

\section{Referencias}

Aguado Terrón,J. M., Feijoo González, C. y Martínez Martínez, I.J. (2013). La comunicación móvil: hacia un nuevo ecosistema digital. Barcelona, España: Gedisa.

Albarello, F. J. (2011). Leer/navegar en internet: las formas de lectura en la computadora. Buenos Aires, Argentina: La Crujía.

Albarello, F. J. (2017). Del contenido al flujo: nuevas relaciones entre medios y usuarios en el ecosistema digital. En C. Labate y C. Arrueta (Eds.), Comunicación digital: redes sociales, nuevas audiencias y 
convergencia. Desafíos y oportunidades para la industria, el Estado y los usuarios. (pp. 219-230). San Salvador de Jujuy, Argentina: Universidad Nacional de Jujuy.

Albarello, F. J. (2019). Lectura transmedia: leer, escribir, conversar en el ecosistema de pantallas. Buenos Aires, Argentina: Ampersand.

Bettetini, G. (1986). La conversación audiovisual. Madrid, España: Cátedra.

Boczkowski, P. J., Mitchelstein, E. \& Matassi, M. (2018). "News comes across when I'm in a moment of leisure": Understanding the practices of incidental news consumption on social media. New Media \& Society, 20(10), 3523-3539. https://doi. org/10.1177/1461444817750396

Burbules, N. (1997). Rhetorics of the web: Hyperreading and critical literacy. En I. Snyder (Ed.), Page to screen: Taking literacy into the electronic era. (pp. 102-122.). New South Wales, Australia: Allen and Unwin.

Castells, M. (1998). La era de la información: economía sociedad y cultura. Vol. 1: La sociedad red. Ciudad de México, México: Alianza.

Chan, J. K. C. \& Leung, L. (2005). Lifestyles, reliance on traditional news media and online news adoption. New Media \& Society, 7(3), 357382. https://doi.org/10.1177/1461444805052281

Chartier, A.-M. y Hébrard, J. (2002). La lectura de un siglo a otro: discursos sobre la lectura (1980-2000). Barcelona, España: Gedisa.

Comscore. (2018). Futuro Digital Global 2018. https://www.comscore.com/lat/Prensa-y-Eventos/Presentaciones-y-libros-blan$\cos / 2018 /$ El-futuro-digital-en-perspectiva

Danno, M. (2018, octubre, 19). We Are Social, the state of the internet in Q4 2018. https://wearesocial.com/au/special-reports/the-state-ofthe-internet-in-q4-2018 
Dimmick, J., Feaster, J. C. \& Hoplamazian, G. J. (2010). News in the interstices: The niches of mobile media in space and time. New Media \& Society, 13(1),23-39. https://doi.org/10.1177/1461444810363452

Dutta-Bergman, M. J. (2004). Complementarity in consumption of news types across traditional and new media. Journal of Broadcasting \& Electronic Media, 48(1), 41-60. https://doi.org/10.1207/ s15506878jobem4801_3

Fernández, J. L. (2018). Plataformas mediáticas: elementos de análisis y diseño de nuevas experiencias. Buenos Aires, Argentina: La Crujía.

Fidler, R. (1998). Mediamorfosis: comprender los nuevos medios. Buenos Aires, Argentina: Granica.

Flanagin, A. J. \& Metzger, M. J. (2001). Internet use in the contemporary media environment. Human Communication Research, 27(1), 153181. https://doi.org/10.1111/j.1468-2958.2001.tb00779.x

Flavián, C. \& Gurrea, R. (2007). Perceived substitutability between digital and physical channels: The case of newspapers. Online Information Review, 31(6), 793-813. https://doi. org/10.1108/14684520710841775

Foltz, P. (1996). Comprehension, coherence, and strategies in hypertext and linear text. En J. F. Rouet, A. Dillon, J. J. Levonen \& R. J. Spiro (Eds.), Hypertext and cognition. (pp. 109-135). Nueva York, EE. UU.: Routledge.

García-Canclini, N. (2007). Lectores, espectadores e internautas. Barcelona, España: Gedisa.

Gentzkow, M. (2007). Valuing new goods in a model with complementarity: Online newspapers. American Economic Review, 97(3), 713744. https://doi.org/10.1257/aer.97.3.713 
Gunter, B., Russell, C., Withey, R. \& Nicholas, D. (2003). The British life and internet project: Inaugural survey findings. Aslib Proceedings, 55(4), 203-216. https://doi.org/10.1108/00012530310486566

Hernández Sampieri, R., Fernández-Collado, C. y Baptista Lucio, M. (2010). Metodología de la investigación. (5. a ed.). Ciudad de México: McGraw-Hill.

Hirst, M. (2011) News 2.0: Can journalism survive the Internet? Crows Newst, Australia: Allen \& Unwin.

Hujanen, J. \& Pietikäinen, S. (2004). Interactive uses of journalism: Crossing between technological potential and young people's newsusing practices. New Media \& Society, 6(3), 383-401. https://doi. org/10.1177/1461444804042521

Igarza, R. (2009). Burbujas de ocio: nuevas formas de consumo cultural. Buenos Aires, Argentina: La Crujía.

Ilhan, B. E., Kozinets, R. \& Otnes, C. (2013). Transmedia consumption experiences (TCE): Patching as a narrative consumption practice. Advances in Consumer Research, 41, 529-531. https: / / www.acrwebsite.org/volumes/1015429/volumes/v41/NA-41

Jenkins, H. (2003, enero 15). Transmedia Storytelling: Moving characters from books to films to video games can make them stronger and more compelling. MIT Technology Review. https://www.technologyreview.com/2003/01/15/234540/transmedia-storytelling/

Jenkins, H. (2008). Convergence culture: la cultura de la convergencia de los medios de comunicación. Barcelona, España: Paidós.

Jenkins, H. (2009). Confronting the challenges of participatory culture: Media education for the 21 st century. Cambridge, RU: The MIT Press. 
Junior,W.(2010).Considerações sobrearelevância dainformaçãojornalística nos sistemas computacionais conectados em rede. Comunicologia: Revistade Comunicação da Universidade Católica de Brasília, 3(2), 14-31. https:// portalrevistas.ucb.br/index.php/RCEUCB/article/view/1911/

Katz, E. (1974). Utilization of mass communication by the individual. En J. G. Blumler \& E. Katz (Eds.), The uses of mass communications: Current perspectives on gratifications research. (pp. 19-32). Beverly Hills, EE. UU.: Sage.

Kayany, J. M. \& Yelsma, P. (2000). Displacement effects of online media in the socio-technical contexts of households. Journal of Broadcasting \& Electronic Media, 44(2), 215-229. https://doi.org/10.1207/ s15506878jobem4402_4

Kaye, B. K. \& Johnson, T. J. (2003). From here to obscurity? Media substitution theory and traditional media in an on-line world. Journal of the American Society for Information Science and Technology, 54(3), 260-273. https://doi.org/10.1002/asi.10212

LaRose, R., Mastro, D. \& Eastin, M. S. (2001). Understanding Internet usage: A social-cognitive approach to uses and gratifications. Social Science Computer Review, 19(4), 395-413. https://doi. org/10.1177/089443930101900401

Lin, C., Salwen, M. B., Garrison, B. \& Driscoll, P. D. (2005). Online news as a functional substitute for offline news. En M. B. Salwen, B. Garrison \& P. D. Driscoll (Eds.), Online news and the public. (pp. 237255). Mahwah, EE. UU.: Lawrence Erlbaum.

Livingstone, S. (2004). The challenge of changing audiences: Or, what is the audience researcher to do in the age of the Internet? European Journal of Communication, 19(1), 75-86. https://doi. org/10.1177/0267323104040695 
Livingstone, S. \& Markham, T. (2008). The contribution of media consumption to civic participation. The British Journal of Sociology, 59(2), 351-371. https://doi.org/10.1111/j.1468-4446.2008.00197.x

Lyons, M. (2012). Historia de la lectura y de la escritura en el mundo occidental. Buenos Aires, Argentina: Editoras del Calderón.

Manovich, L. (2013). Software takes command. Nueva York, EE. UU.: Bloomsbury Academic.

Márquez, I. (2015). Una genealogía de la pantalla: del cine al teléfono móvil. Barcelona, España: Anagrama.

Martini, S. y Luchessi, L. (2004). Los que hacen las noticias: periodismo, información y poder. Buenos Aires, Argentina: Biblos.

McLuhan, M. (1994). Comprender los medios de comunicación: las extensiones del ser humano. Barcelona, España: Paidós.

McQuail, D. (1969). Sociología de los medios masivos de comunicación. Barcelona, España: Paidós.

Mitchelstein, E. \& Boczkowski, P. J. (2010). Online news consumption research: An assessment of past work and an agenda for the future. New Media \& Society, 12(7), 1085-1102. https://doi. org/10.1177/1461444809350193

Mora, V. L. (2012). El lectoespectador: deslizamientos entre literatura e imagen. Barcelona, España: Seix Barral.

Nguyen, A. \& Western, M. (2007). Socio-structural correlates of online news and information adoption/use: Implications for the digital divide. Journal of Sociology, 43(2), 167-185. https://doi. org/10.1177/1440783307076894 
Ong, W.J. (1994). Oralidad y escritura: tecnología de la palabra. Bogotá, Colombia: Fondo de Cultura Económica.

Postman, N. (1992). Technopoly: The surrender of culture to technology. Nueva York, EE. UU.: Random House.

Reuters Institute. (2018). Digital Report 2018. Recuperado de shorturl. at/dktHQ

Scolari, C. (2004). Hacer clic: hacia una sociosemiótica de las interacciones digitales. Barcelona, España: Gedisa.

Scolari, C. (2013). Narrativas transmedia: cuando todos los medios cuentan. Barcelona, España: Deusto.

Scolari, C. (Ed.) (2015). Ecología de los medios: entornos, evoluciones e interpretaciones. Barcelona, España: Gedisa.

Scolari, C. (2016). El translector: lectura y narrativas transmedia en la nueva ecología de la comunicación. En J. A. Millán (Coord.), La lectura en España: Informe 2017. (pp. 175-186). Madrid, España: Federación de Gremios de Editores de España.

Scolari, C. (2018). Las leyes de la interfaz: diseño, ecología, evolución, tecnología. Barcelona, España: Gedisa.

Srniceck, N. (2018). Capitalismo de plataformas. Buenos Aires, Argentina: Caja Negra.

Sheehan, K. B. (2002). Of surfing, searching, and newshounds: A typology of Internet users' online sessions. Journal of Advertising Research, 42(5), 62-71. https://doi.org/10.2501/JAR-42-5-62-71

Shirky, C. (2008). Here comes everybody: Revolution doesn't happen when society adopts new technology, it happens when society adopts new behaviors. Londres, RU: Penguin Books. 
Taneja, H., Webster, J. G., Malthouse, E. C. \& Ksiazek, T. B. (2012). Media consumption across platforms: Identifying user-defined repertoires. New Media \& Society, 14(6), 951-968. https://doi. org/10.1177/1461444811436146

Valles Martínez, M. S. (1999). Técnicas cualitativas de investigación social: reflexión metodológica y práctica profesional. Madrid, España: Síntesis.

Van Dijck, J., Poell, T. \& De Waal, M. (2018). The platform society: Public values in a connective world. Nueva York, EE. UU.: Oxford University Press.

Van Dijk, T. (1989). La ciencia del texto. Barcelona, España: Paidós.

Wimmer, R. D. y Dominick, J. R. (1996). La investigación cientifica de los medios de comunicación: una introducción a sus métodos. Barcelona, España: Bosch. 